\title{
Longitudinal study of a community hospital integrated model for diabetes management in the Beijing Jingsong community
}

\author{
Jiandong Zhang ${ }^{1}$, Ning Zhuang ${ }^{1}$, Mingxia Yuan ${ }^{3}$, Lijuan Gao ${ }^{1}$, Chuan Bian ${ }^{1}$, Huaining Chen $^{1}$, Qing Chi ${ }^{1}$, Suqing Hui ${ }^{1}$,
} Guiying $\mathrm{Li}^{1}$, Yanli $\mathrm{Su}^{1}$, Hongsong $\mathrm{Bao}^{1}$, Gang Wan ${ }^{2}$, Guangran Yang ${ }^{3}$, Shenyuan Yuan ${ }^{3}$

\begin{abstract}
Objective: To determine the effects of a community hospital integrated model on the longitudinal management of diabetic patients.

Methods: Four hundred forty-one patients with type 2 diabetes residing in Jingsong Community were randomly assigned to intensive and standard groups. Metabolic parameters were measured in the two groups at baseline and after 36 months of management to compare the rate of goal achievement.

Results: After comprehensive management, the overall control rate in the intensive group was increased from $2.7 \%$ to $9.6 \%$ compared with $2.3 \%-4.5 \%$ in the standard group. Specifically, the control rates for fasting plasma glucose, glycosylated hemoglobin, and blood pressure in the intensive group were significantly increased from $54.1 \%, 40.0 \%$, and $68.2 \%$ to $85.5 \%, 74.6 \%$, and $89.1 \%$, respectively. The control rate for glycosylated hemoglobin in the intensive group was significantly higher than the standard group after 36 months of treatment.

Conclusion: The community hospital integrated model for longitudinal management effectively improved the control rate of glycosylated hemoglobin and the overall control rate in patients with type 2 diabetes.
\end{abstract}

Keywords: Diabetes, Community management, Overall control

\section{Introduction}

Diabetes is a chronic, lifelong disease associated with a number of potential complications. Diabetic complications can be disabling and are the main cause of death. Diabetes and its complications cause an enormous economic burden to affected families and society. Intensive glucose control in patients with type 2 diabetes (hereafter referred to as diabetes) can prevent the development and progression of microvascular complications. The United Kingdom Prospective Diabetes Study
(UKPDS) showed that compared with the control group, intensive glucose control had no significant effect on the risk of myocardial infarction $[1,2]$. During the extended period of the study, however, the risks for diabetesrelated endpoints were decreased, and the risks for myocardial infarction and all-cause death were decreased by $13 \%$ [3]. A metaanalysis showed that the relative risk for nonfatal myocardial infarction is reduced by $15 \%$ when the glycosylated hemoglobin (HbAlc) level is decreased by $1 \%$ [4]. Between $70 \%$
1. Jingsong Community Health Service Center, Beijing 100021, China

2. Medical Record Statistics Room, Beijing Ditan Hospital, Capital Medical University, Beijing 100015, China

3 Endocrinology Department, Beijing Tongren Hospital, Capital Medical University, Beijing 100730, China

CORRESPONDING AUTHORS: Shenyuan Yuan, Guangran Yang, Endocrinology Department, Beijing Tongren Hospital, Capital Medical University, Beijing 100730, China

E-mail: fulvic@126.com,

yanggr_55@126.com

Funding: This project [ST12024] is supported by a BRIDGES Grant from the International Diabetes Federation (BRIDGES, an International Diabetes Federation project, is supported by an educational grant from Lilly Diabetes.), and a Grant of Special Scientific Research on Capital Health Development [2011-2005-01].

Received 12 December 2013; Accepted 20 February 2014 
and $80 \%$ of patients with type 2 diabetes die of cardiovascular diseases. To decrease the overall rate of diabetic micro- and macro-vascular complications, comprehensive management must be implemented for diabetic patients residing in communities, which has become an important objective for reducing the threat of diabetes. As one of the main battlefields for prevention and treatment of diabetes in Beijing, our Center began to cooperate with Class Three Grade A hospitals in 2008, and has since carried out a joint comprehensive management program for diabetics residing in communities for 36 months. The aim of the current study was to determine various metabolic indices before and after management to highlight changes in the overall control rate and to identify a management pattern suitable for diabetics residing in communities.

\section{Subjects and methods}

Subjects

The current study was a sub-program of the longitudinal diabetes management program (Beijing Community Diabetes Study) that was jointly carried out by Class Three Grade A hospitals and 15 urban communities in Beijing. Fifteen community health centers in Beijing were selected using a multistage random sampling method [5]. People with type 2 diabetes (20-80 years of age) who lived in Jingsong Community $>5$ years were recruited for the study between August 2008 and July 2009. The exclusion criteria were as follows: (1) patients with a high level of residential mobility, poor compliance, and serious disability; (2) patients with hepatic or renal failure; and (3) patients with serious schizophrenia. A total of 441 patients were enrolled in this study for management. Using a random number table, the patients were randomly assigned to an intensive management group ( $n=220$ patients) and a standard management group ( $n=221$ patients). The management period was 36 months. The authors received consent from all participants.

\section{Methods}

Multi-center study on the community hospital integrated model for diabetes management: Under the guidance of chief physicians from Class Three hospitals during the whole process, and according to the Chinese Guideline for Type 2 Diabetes in 2007 [6], management regulations were formulated for each group and general practitioners responsible for the management work were designated. In the intensive group, HbAlc measurement was performed every 3 months and the urinary albumin excretion rate (UAER) measurement was performed every 6 months. Follow-up visits and medical record collection were performed every 2 months in the intensive group. In the standard group, HbAlc measurement was performed every 6 months and UAER measurement was performed yearly. Follow-up visits and medical record collection were performed every 3 months in the standard group. Hepatic and renal function, lipid profiles, and electrocardiograms were performed for all patients yearly. Retinal photography was also performed annually for all patients to screen for retinopathy.

Evaluation indexes: Physical examinations were performed. Physiologic and biochemical indices were measured. The control rates were calculated.

Patient transfer indications: Patients with poor glycemic control, acute serious metabolic disorders, infectious complications, or severe and complex chronic complications were therefore transferred from the Community Health Service Center to Class Three Grade A hospitals. When such conditions were controlled and stabilized, the patients were transferred back to the Community Health Service Center from Class Three Grade A hospitals.

Target goals for clinical measures were based on the Chinese Guideline for Type 2 Diabetes, [6, 7]: Fasting plasma glucose (FPG): FPG $<7.2 \mathrm{mmol} / \mathrm{L}$; HbAlc $<7.0 \%$; systolic blood pressure (SBP) $<130 \mathrm{mmHg}$; diastolic blood pressure (DBP) $<80 \mathrm{mmHg}$; low density lipoprotein-cholesterol (LDL-c): $<2.6 \mathrm{mmol} / \mathrm{L}$ for patients without coronary heart disease, $<1.8$ $\mathrm{mmol} / \mathrm{L}$ for patients with coronary heart disease. Overall control rates: The calculation included patients who met all these goals.

Statistical analysis: The database was established using EpiData3.0, and specially-assigned personnel were appointed to verify data entries and import the data to SPSS 11.5. Then, various data of enrolled patients before and after 36 months of treatment were processed and analyzed. Continuous variables were assessed by a t-test and expressed as the mean and 
standard deviation. Enumeration variables were assessed by a $\chi^{2}$ test and expressed as a percentage. A $P$ value $<0.05$ in twotailed tests was considered to be statistically significant.

\section{Results}

\section{General conditions of patients with diabetes}

Four hundred forty-one patients were between 30 and 87 years of age. The average ages were $64.7 \pm 8.4$ years and $64.2 \pm 9.0$ years in the intensive and standard groups, respectively $(P>0.05)$. Most patients were women, accounting for $64.5 \%$ and $67.4 \%$ of the participants in the intensive and standard groups, respectively. The Han population accounted for the vast majority of participants (97.2\% and $96.3 \%$, respectively). Married patients accounted for $98.6 \%$ and $97.8 \%$ of the participants, respectively. Most of the patients were retired $(81.7 \%$ and $78.4 \%$, respectively). The monthly incomes were 1000 4000 yuan for most families, accounting for $90.9 \%$ and $96.4 \%$ of the participants, respectively. The average duration of disease was 8.4 \pm 7.1 years and 8.0 \pm 7.2 years in the intensive and standard groups, respectively. There were no significant differences in clinical characteristics between the two groups $(P<0.05)$.

\section{Comparison of control rates of blood glucose, blood pressure, and blood lipids}

Before management, the differences in the FPG, HbA1c, blood pressure, LDL-c, and overall control rate in the intensive and standard group were not statistically significant. After management, the FPG, HbA1c, blood pressure, and overall control rate of both groups were increased $(P<0.05)$. The control rates of various indices in the intensive group were higher than the standard group. The overall control rate in the intensive group after treatment was significantly higher than the standard group $\left(\chi^{2}=4.522 P=0.039\right)$. The FPG, HbA1c, SBP, DBP, and LDL-c in the intensive and standard groups were significantly decreased compared to before treatment. The overall control rate in the standard group was increased after management; however, the difference was of no statistical significance (Tables 2 and 3, Fig. 1).

Table 1. The demographic characteristics between the intensive and standard groups.

\begin{tabular}{|c|c|c|c|c|}
\hline Characteristic & $\begin{array}{l}\text { Intensive group } \\
(n=220)\end{array}$ & $\begin{array}{l}\text { Standard group } \\
(n=221)\end{array}$ & Statistics & $P$-value \\
\hline Gender & & & 0.406 & 0.524 \\
\hline Male & $78(35.5)$ & $72(32.6)$ & & \\
\hline Female & $142(64.5)$ & $149(67.4)$ & & \\
\hline Age (years) & $64.7 \pm 8.4$ & $64.2 \pm 9.0$ & $0.577^{\mathrm{a}}$ & 0.564 \\
\hline Nationality & & & 0.921 & 0.418 \\
\hline Han & 214(97.3) & 213(96.4) & & \\
\hline Others & $6(2.7)$ & $8(3.6)$ & & \\
\hline Marital status & & & 7.875 & 0.096 \\
\hline Married & 201(91.4) & $185(83.7)$ & & \\
\hline Others & 19(8.6) & $36(16.3)$ & & \\
\hline Occupation & & & 0.864 & 0.353 \\
\hline Retired & $180(81.8)$ & $173(78.3)$ & & \\
\hline Others & $40(18.2)$ & $48(21.7)$ & & \\
\hline Family incomes (yuan) & & & 9.759 & 0.135 \\
\hline$<1000$ & $5(2.3)$ & $4(1.8)$ & & \\
\hline $1000 \sim 4000$ & $117(53.2)$ & $124(56.1)$ & & \\
\hline$>4000$ & $98(44.5)$ & $93(42.1)$ & & \\
\hline Duration of diabetes (years) & $8.4 \pm 7.1$ & $8.0 \pm 7.2$ & $0.546^{\mathrm{a}}$ & 0.585 \\
\hline
\end{tabular}

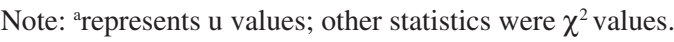




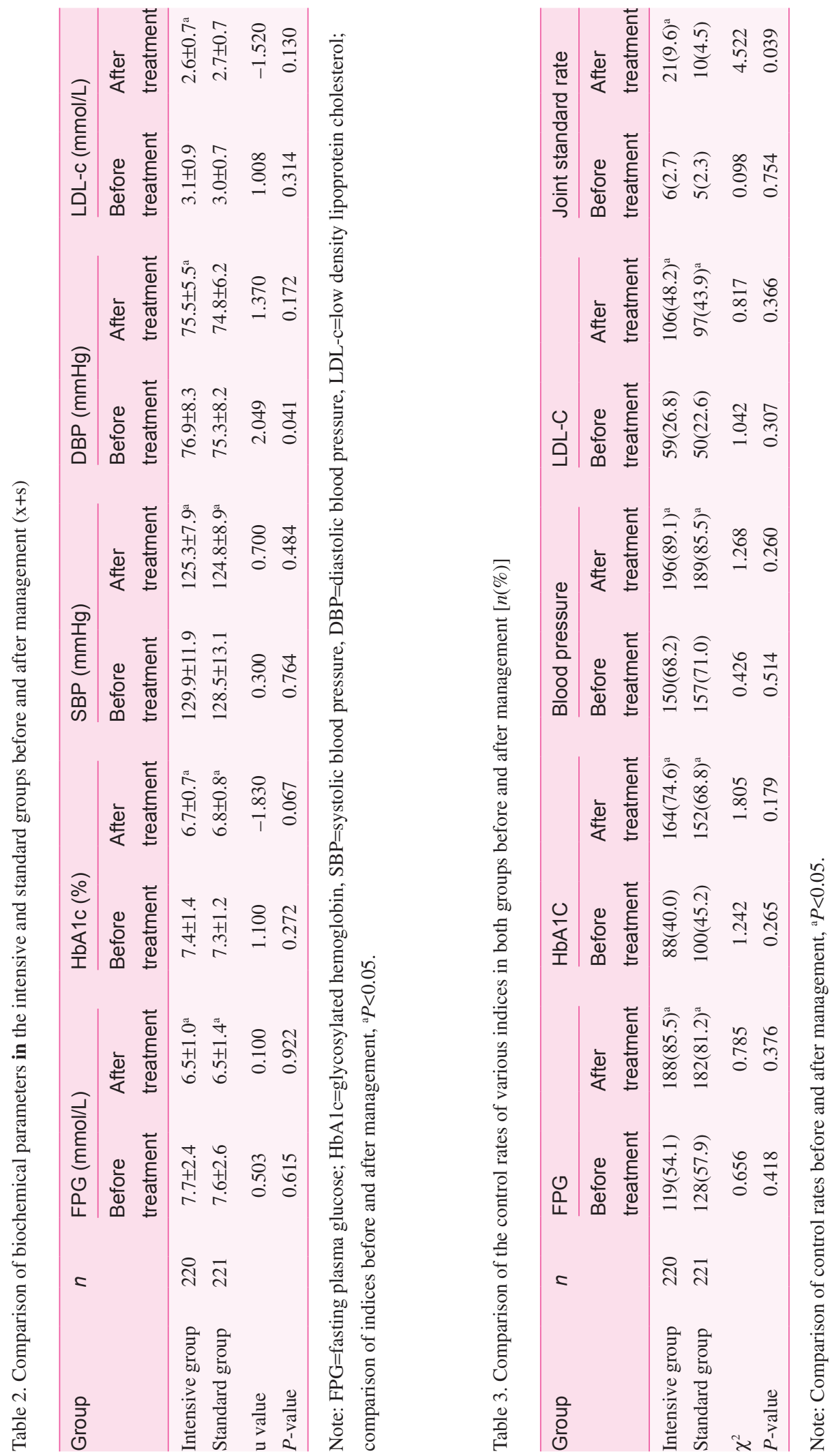



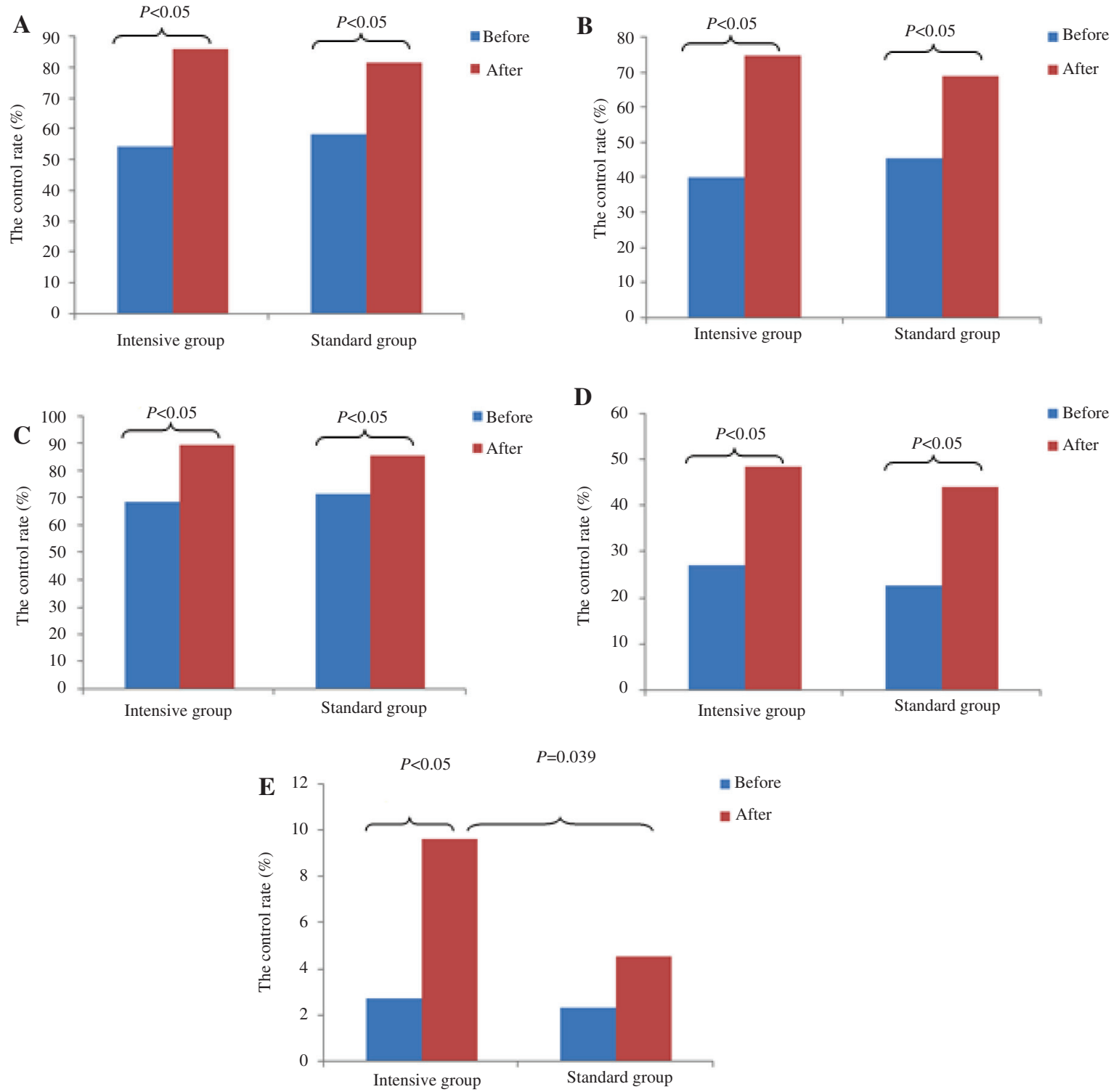

Fig. 1. The control rates of fasting plasma glucose (A), HbA1c (B), blood pressure (C), low density lipoprotein (D), and the overall control rate (E) in both groups before and after management.

\section{Discussion}

Diabetes is a clinical syndrome with metabolic disorders as the main manifestation and vascular complications as the main outcome. Only by achieving overall control of various risk factors for diabetes can the progression of diabetes and occurrence of complications be controlled [1,2]. Although the professional technical strength of Class Three and Class Two 
that diabetes management in communities will improve the treatment effects [11-14]. Therefore, the community hospital integrated model for diabetes management emerged, and its remarkable effects had been confirmed in practice. Internetbased video case consultation between general practitioners and specialists was used in one study [15]. In the current study, chief physicians from Class Three Grade A hospitals were directly assigned to communities. The chief physicians provide guidance, training, and face-to-face communication with general practitioners and patients, thus improving the management level of diabetes, resident health awareness, and good compliance in communities, helping the patients maintain a reasonable diet, appropriate exercises, and medications.

Health records had been filed in the Community Health Service Center for the 441 diabetic patients that voluntarily participated in this study and the long-term follow-up treatment. After 36 months of standardized management, especially the community hospital integrated model for diabetes management, the patients' mean glucose level was decreased to 6.5 $\mathrm{mmol} / \mathrm{L}$ and the mean $\mathrm{HbAlc}$ was decreased to $6.7 \%$. The rate achieving $\mathrm{HbAlc}<7.0 \%$ in the intensive group was increased from $40 \%$ before treatment to $74.6 \%$ after treatment compared to the $68.8 \%$ of the standard group after treatment. Chi et al. [16] reported the level of glucose control for 2077 inpatients and outpatients with diabetes from 36 hospitals of Beijing in 2008; $24.6 \%$ of the patients achieved a HbAlc $<6.5 \%$. In the BCDS study [17] involving 15 communities in Beijing, 31.8\% of patients with type 2 diabetes achieved a HbA1c $<6.5 \%$. Approximately $30 \%$ of patients with diabetes achieve euglycemia, which may be improved with management.

Management of diabetes is not only to ensure blood glucose, but also other various risk factors under control, such as blood lipids and blood pressure. Management of diabetes includes individualized guidance of lifestyle. Based on the UKPDS, intensive blood pressure control reduced the mortality and risk of related complications in diabetic patients with hypertension [18]. In the Beijing communities, however, $17.8 \%$ of patients with type 2 diabetes were normotensive [19] and $30.9 \%$ of patients with dyslipidemia had LDL-c under control [20]. For patients in the intensive group, the SBP and DBP were decreased by $4.6 \mathrm{mmHg}$ and $1.4 \mathrm{mmHg}$, respectively, which was significantly different compared with before treatment. For patients in the standard group, SBP was decreased by $3.7 \mathrm{mmHg}$; however, DBP was reduced by only $0.5 \mathrm{mmHg}$ (no significant difference). LDL-c in the intensive group was significantly decreased, while the change in LDL-c in the standard group was not significantly different.

According to the recommendations of the China Guideline for Type 2 Diabetes in 2007 and 2010, management goals may only be achieved with comprehensive management, and the effects of comprehensive management of diabetes can only be explained through overall control of related measures. As shown in the current study, the community hospital integrated model for diabetes management increased the overall control rate of the 441 patients. After treatment, the overall control rate of the intensive group was significantly increased by $6.9 \%$ (from $2.7 \%$ to $9.6 \%$ ), and the overall control rate in the standard group was increased by $2.2 \%$ (no significant difference). The American Diabetes Association (ADA) and Chinese Diabetes Society have both recently emphasized the importance of overall control in diabetes management $[7,21]$. The overall control standard in the current study was designed according to the recommendations of the Chinese Guideline for Type 2 Diabetes 2010 to further prove the outstanding effects of this pattern in comprehensive prevention and treatment for diabetics in communities. There is a large number of diabetic patients in need of management within communities; however, general practitioners lack public confidence and influence due to their limited technical level. Thus, the longterm consulting arrangement undertaken by experts from Class Three Grade A hospitals provided community patients with an expert level of service comparable to tertiary hospitals, and a cohort of physicians with continuously improving clinical skills. The entire medical strength of communities and patient compliance with diabetic management were also gradually improved, which in turn proved the effectiveness of the community hospital integrated model for diabetes management in communities. With lower costs and better effects, the BCDS has demonstrated a fine example of detailed management, which is a suitable pattern worthy of implementation among other communities.

In addition, as shown in the current study, in the intensive group the improvement in lipid control was not as significant as control of blood glucose and blood pressure. This finding may be related to general practitioners paying more attention to blood glucose and blood pressure during the management 
process. Alternatively, the finding also indicates that general practitioners should pay more attention to lipid control. Then, the concept could be transferred to the patients, thus achieving a better overall control outcome. Therefore, this management pattern should be further improved based on future studies.

\section{Contributions of authors}

Jiandong Zhang: Had a leadership role in all studies and most of the scientific research design, and assisted in completion of a portion of the manuscript.

Ning Zhuang: Had a leadership role in most studies and collecting and managing the main scientific research data, and assisted in completion of a portion of the manuscript.

Mingxia Yuan: Responsible for most of the scientific research design.

Lijuan Gao: Responsible for statistics of some of the data.

Chuan Bian, Huaining Chen, Qing Chi, Suqing Hui, Guiying Li, Yanli Su, and Hongsong Bao: Responsible for longitudinal management of some of the patients.

Gang Wan: Responsible for statistics of the main data.

Shenyuan Yuan: Responsible for scientific research design and assisting in completion of the manuscript.

Guangran Yang: Responsible for a portion of the scientific research design and assisting in completion of the manuscript.

\section{Conflict of interest}

The authors declare no conflict of interest.

\section{References}

1. Pan C. New viewpoints on comprehensive management of diabetes. China Med Tribune 2008;34:6.

2. Stratton IM, Adler AI, Neil HA, Matthews DR, Manley SE, Cull CA, et al. Association of glycaemia with macrovascular and microvascular complications of type 2 diabetes (UKPDS 35): prospective observational study. Br Med J 2000;321:405-12.

3. Holman RR, Paul SK, Bethel MA, Matthews DR, Neil HA. 10year follow-up of intensive glucose control in type 2 diabetes. $\mathrm{N}$ Engl J Med 2008;359:1577-89.

4. Turnbull FM, Abraira C, Anderson RJ, Byington RP, Chalmers JP, Duckworth WC, et al. Intensive glucose control and macrovas- cular outcomes in type 2 diabetes. Diabetologia 2009;52:228898.

5. Xu J, Wei WB, Yuan MX, Yuan SY, Wan G, Zheng YY, et al. Prevalence and risk factors for diabetic retinopathy: the Beijing Communities Diabetes Study 6. Retina 2012;32:322-9.

6. Xiang K, Yang W. China Guideline for Type 2 Diabetes (2007). 2007.

7. China Diabetes Society (CDS). China Guideline for Type 2 Diabetes. China Diabetes 2010;2 Suppl 2:1-56.

8. Satterfield DW, Volansky M, Caspersen CJ, Engelgau MM, Bowman BA, Gregg EW, et al. Community-based lifestyle interventions to prevent type 2 diabetes. Diabetes Care 2003;26:2643-52.

9. Shah M, Kaselitz E, Heisler M. The role of community health workers in diabetes: update on current literature. Curr Diab Rep 2013;3:163-71.

10. Zhou Y, Cheng J, Zhao L, Sheng HG, Chen L, Zhang XY, et al. Diabetes control in hospitals at different levels in Shanghai, and living habits and self-management conditions. Shanghai Med J 2009;32:602-6.

11. Chang S, Zhu W, Dong S. Study on management pattern of diabetes in communities. Chinese J Curr Clin Med 2005;4:311-3.

12. Jiang $\mathrm{S}$. New strategies for conducting diabetes health education and health promotion in communities. Chinese General Pract 2009; $12: 1558$

13. Ruan XN, Fu DP, Fu H. Effect assessment of health education for diabetics' self-management in Pudong New Area of Shanghai. Chinese General Pract 2009;12:1400.

14. Henderson VA, Barr KL, An LC, Guajardo C, Newhouse W, Mase R, et al. Community-based participatory research and usercentered design in a diabetes medication information and decision tool. Prog Community Health Partnersh 2013;7:171-84.

15. Zhao LB, Chen YH, Dong B, Li YD, Zhou YX, Lu L, et al. A new model for diabetes care based on GPs-specialists cooperation through internet in community: Shanghai Wuliqiao study. Chin J Endocrinol Metab 2012;28:286-9.

16. Chi JM, Wu Q, Qin MZ, Guo XP, Zhu SN, Yao C. The investigation of treatment target for 2077 patients with type 2 diabetes mellitus in Beijing. Beijing Medical Journal 2008;30:513-5.

17. Yuan M, Yuan S, Fu H, Wan G, Zhu LX, Zhang MX, et al. Current control status of glycolated hemoglobin level for community patients with type 2 diabetes in Beijing and the influencing factors - Beijing community diabetes study BCDS-3. Chinese J Diabetes 2010;(10):752-5.

18. UK Prospective Diabetes Study Group. Tight blood pressure control and risk of macrovascular and microvascular complica- 
tions in type 2 diabetes: UKPDS 38. Br Med J 1998;317:703-13 and 1999;318:29.

19. Qin M, Yuan S, Fu H, Wan G, Zhu LX, Zhang MX, et al. Survey on the rate and blood pressure standard of patients with type 2 diabetes and complicated with hypertension in 15 communities in Beijing. Chinese J Gen Prac 2013;12:537-40.
20. Qin M, Yuan S, Fu H, Wan G, Zhu LX, Yuan MX, et al. Analysis on blood fat disorders of patients with type 2 diabetes in communities in Beijing - Beijing Community Diabetes Study (BCDS8). Chinese J Gen Prac 2012;11:748-52.

21. American Diabetes Association, Standards of medical care in diabetes - 2007. Diabetes Care 2007;30 Suppl 1:4-41. 